\title{
Aprendizaje basado en proyectos soportado en un diseño tecno-pedagógico para la enseñanza de la estadística descriptiva
}

\author{
Javier D. Vargas ${ }^{1}$, Isabel C. Arregocés ${ }^{1}$, Andrés D. Solano ${ }^{1}$ y Katia K. Peña ${ }^{2}$ \\ (1) Fac. Ciencias Económicas y Administrativas, Universidad de La Guajira, Km 5 vía Maicao, Riohacha- La Guajira- \\ Colombia (correo-e: jvargas@uniguajira.edu.co) \\ (2) Fac. Ciencias de la Educación, Universidad de La Guajira, Km 5 vía Maicao, Riohacha- La Guajira-Colombia; \\ (correo-e: icarregoces@uniguajira.edu.co, andresolano@uniguajira.edu.co,kpena@uniguajira.edu.co)
}

Recibido Mar. 17, 2021; Aceptado May. 14, 2021; Versión final Jul. 8, 2021, Publicado Dic. 2021

\begin{abstract}
Resumen
El principal propósito de esta investigación es analizar como la incorporación de la estrategia pedagógica de aprendizaje basada en proyectos permite fortalecer la enseñanza de estadística descriptiva en el programa de negocios internacionales de la Universidad de La Guajira (Colombia). La metodología incluye una observación directa a 21 estudiantes, análisis de contenido y análisis estadístico de registros. Los resultados muestran que las actividades que integran las tecnologías de la información y comunicación (TIC) permiten un mayor dominio a los estudiantes en el manejo de datos estadísticos contextualizados. El aprendizaje basado en proyectos (ABPy), con un diseño tecno-pedagógico, permite que los estudiantes relacionen contenidos teóricos con la práctica, posibilitando el desarrollo de competencias estadísticas, comunicativas, digitales y de pensamiento crítico. Se concluye que con la implementación de la metodología de aprendizaje basada en proyectos se potencia la construcción de conocimiento en estadística y se fortalece la habilidad para realizar análisis estadísticos.
\end{abstract}

Palabras clave: diseño tecno-pedagógico; proyectos; aprendizaje; TIC; estadística descriptiva

\section{Project-based learning supported by a techno-pedagogical design for teaching descriptive statistics}

\begin{abstract}
The primary purpose of this research is to examine whether implementing a pedagogical project-based learning strategy strengthens teaching of descriptive statistics in the international business program at the Universidad de la Guajira (Colombia). The methodology includes direct observation of 21 students, content analysis, and record statistical analyses. The results show that the activities that integrated information and communication technologies (ICTs) improve student management of contextual statistical data. Applying project-based learning (PBL), having a techno-pedagogical design, enhances correlating theoretical content to practical content, which promotes the development of statistical, communicative, digital, and critical thinking skills. It is concluded that implementing a project-based learning strategy boosts student knowledge on statistics while strengthening statistical analysis skills.
\end{abstract}




\section{INTRODUCCIÓN}

En la actualidad las universidades siguen implementando acciones con el principal propósito de producir aprendizajes consolidados y profundos en los estudiantes, para que esto incida favorablemente en la calidad académica, especialmente en el rendimiento académico de los mismos. Algunas investigaciones han argumentado diversos factores de riesgo que afectan el desempeño académico y estos factores pueden ser socioeconómicos, académicos, institucionales e individuales (Gutiérrez-Monsalve, et al., 2021). Dichos factores conforman unas de las principales variables asociadas al bajo rendimiento académico de los estudiantes universitarios. (Souza, et al., 2017). En ese sentido, dichas variables deben ser tenidas en cuenta a la hora de la planificación y uso de nuevas estrategias para la enseñanza al interior de las aulas.

En América Latina, específicamente en el caso de Colombia, los análisis estadísticos y cualitativos de las diferentes universidades tanto públicas como privadas, indican que el bajo rendimiento académico sigue siendo una situación por superar (Souza et al., 2017). En las universidades de la Costa Caribe de Colombia, el bajo rendimiento académico se observa en los resultados de las competencias matemáticas, como lo evidencian los resultados de las pruebas "Saber Pro" del ICFES (Instituto Colombiano para el fomento de la Educación Superior) aplicadas a los estudiantes universitarios próximos a titularse. La aplicación de estas pruebas en el 2013 a una muestra de estudiantes de la carrera de Negocios Internacionales de la Facultad de Ciencias Económicas y Administrativas de la Universidad de la Guajira determinó deficiencias en el área referente al razonamiento cuantitativo, debido a que el $66,7 \%$ de la muestra de estudiantes obtuvo un nivel muy bajo en esta área del conocimiento. Estas debilidades en las competencias de razonamiento cuantitativo afectan negativamente el aprendizaje de la asignatura de Estadística I (descriptiva). En la Universidad de la Guajira, los estudiantes en los programas de Ciencias Económicas y Administrativas evidencian un débil desempeño en las competencias matemáticas, teniendo en cuenta que la asignatura Estadística I tiene un alto contenido de estas competencias, el estudio realizado por Vargas (2018) revela que desde el año 2013 al 2015, el promedio de pérdida de la asignatura Estadística descriptiva es de un $49,3 \%$. Por lo que se hizo necesaria la implementación de una estrategia pedagógica basada en proyectos con el apoyo de las TIC, en la mencionada asignatura del referido programa de Negocios Internacionales en la Universidad de La Guajira.

El Aprendizaje Basado en Proyectos (ABPy) como una estrategia pedagógica, que según investigadores como (Villalobos-Abarca, et al., 2018; Dierker, et al., 2018), busca incluir en el desarrollo de los contenidos programáticos, la realización de proyectos que permitan la integración de la teoría con la práctica y el trabajo colaborativo, reflejando la aplicabilidad de los conocimientos adquiridos de las diferentes áreas de la formación académica y los obtenidos por la experiencia del estudiante. Es en ese mismo sentido, que Kokotsaki et al, 2016, consideran que esta estrategia es una forma de instrucción que centra su atención en el aprendizaje de los estudiantes, específicamente en la aplicación de conocimientos en contextos específicos donde los estudiantes participan activamente por medio de las interacciones sociales desde lo colectivo. Por su parte, sostienen Acharya y Gayana (2021) que, con esta estrategia pedagógica cambia el rol al docente en un facilitador y permite la utilización de herramientas tecnológicas que medien los procesos de enseñanza y de aprendizaje; el estudiante estará motivado debido a que trabaja sobre sus propias ideas, maneja sus tiempos, integra los aprendizajes de aula con la realidad, desarrolla habilidades de solución de problemas ( Rodríguez, Ordoñez y Meneses, 2018), y toma de decisiones, situaciones o elementos que son importantes para la Estadísticas I.

El ABPy se desarrolla tomando como base los principios del modelo pedagógico constructivista con esta estrategia de enseñanza se busca que los estudiantes construyan conocimiento a partir de actividades teórico-prácticas y tengan con ello un aprendizaje activo (Clark, et al., 2020; Veselov, et al., 2019). Es así como el estudiante debe asumir responsabilidades en cuanto a su aprendizaje, trabajar colaborativamente con sus compañeros, compartir ideas, información, ser autónomo en su aprendizaje y diseñar estrategias para la planificación, control y evaluación del proceso de aprendizaje. Para realizar el trabajo colaborativo entre los estudiantes, se recurre a la clasificación por sus estilos/entradas de aprendizajes, usando del modelo VAK (Visual, Auditivo y Kinestésico), que pueden entenderse características psicológicas, rasgos cognitivos, afectivos y fisiológicos que posee una persona para enfrentarse a una situación de aprendizaje, tal como lo plantea Seitova et al., 2016.

Para atender la necesidad encontrada en el aula, se hace una integración de la pedagogía y la tecnología para generar una combinación que sirve para concebir un diseño tecnopedagógico (Yeh et al., 2017). En ese sentido, el diseño tecnopedagógico puede entenderse como un proceso sistemático para planificar la enseñanza (Leung, 2017). Por su parte, Solano y Aarón, (2020) citando a Coll et al., (2007, p. 380) muestran como desde el diseño tecnopedagógico se busca integrar "los aspectos tecnológicos como los pedagógicos o instruccionales y que adoptan la forma de un diseño tecnopedagógico o tecnoinstruccional". Teniendo en cuenta lo anterior, para los autores de esta experiencia este diseño tecnopedagógico fue 
pensado desde un componente pedagógico empleando la estrategia de aprendizaje basado en proyectos para atender a los objetivos de enseñanza, y se usaron como mediaciones tecnológicas algunas TIC, explicadas más adelante, que permitieron atender las dificultades de aprendizaje encontradas en el aula.

En cuanto a la incorporación de las TIC para apoyar el diseño tecnopedagógico, se utilizó la mediación de un curso en modalidad mixta (presencial y virtual), alojado en una plataforma virtual educativa, como lo fue la plataforma Virtual Akumaja de la Universidad de La Guajira, soportada en Moodle, este tipo de recurso tecnológico ha recibido diferentes denominaciones, entre las más comunes se encuentran el aula virtual, plataforma virtual de aprendizaje y entorno virtual de enseñanza y aprendizaje (EVE/A). La mediación de las herramientas tecnológicas puede ser de gran aporte en la medida de que faciliten el desarrollo de las actividades, que permita suministrar al estudiante los materiales y recursos oportunos y valiosos en el desarrollo de la estrategia pedagógica, así como mantener un constante manejo y control de la actividad extractase.

Entre las herramientas tecnológicas, se destacan principalmente los sitios Web, foros virtuales, videoconferencias, los chats académicos, la mensajería instantánea, los videos tutoriales y el correo electrónico, los cuales de forma sistemática permiten a los equipos realizar el trabajo que deben llevar a cabo. Con respecto a los videotutoriales, estos, en educación facilita la atención personalizada al estudiante, propiciando que el aprendizaje sea autónomo y con muchas posibilidades de ser significativo. En cuanto a los foros virtuales como recurso asíncrono, se constituyen en un espacio apto para la promoción de comportamientos colaborativos entre los estudiantes, que permite que cada participante reconozca los aportes de los demás, reflexionen sobre ellos y construyan sus aportes de acuerdo con su propio ritmo de aprendizaje. De igual manera, se utilizaron en el desarrollo de los proyectos herramientas ofimáticas como Microsoft Word el procesador de palabras (textos), Microsoft Power Point para la elaboración de presentaciones de los proyectos y las hojas de cálculo de Microsoft Excel (Bueno, 2017). La hoja de cálculo de Microsoft Excel se selecciona para la enseñanza la estadística descriptiva por la facilidad del uso y porque "se ha convertido en un software estándar en los entornos de enseñanza, profesionales y familiares. Excel tiene una interfaz amigable y es fácil usar" (Cao y Naya, 2010, p.2)

\section{OTROS ANTECEDENTES}

La implementación del ABPy se encuentran en diferentes contextos de educación superior como en las ingenierías (Cruz, Serrano y Rodríguez, 2021; Burgos-Leiva et al., 2021; Herrera, 2017), en la enseñanza de la medicina veterinaria y biología (Mateos et al, 2020), en cursos de geología (Rodríguez-Oroz et al, 2019), en educación y pedagogía (Ausín, et al., 2016). Cada uno de esos escenarios de enseñanza y aprendizaje ha considerado la inclusión de esta estrategia pedagógica porque facilita la comprensión y apropiación de los temas que son abordados en las diferentes asignaturas. Por su parte, en el tema de estadística también existen algunos referentes en el uso de esta estrategia del ABPy para facilitar la comprensión de ella en situaciones contextualizadas (Flores y Pinto, 2017; Batanero et al., 2013; Alvarado, Galindo y Retamal, 2013). Entre los antecedentes sobre diseño tecnopedagogico de esta de investigación se encuentran algunos diseños acompañados de pedagogía y tecnología para potenciar el proceso de enseñanza, entre ellos se puede referenciar a Solano y Aarón (2020) con su artículo de "enseñanza en ingeniería de manera colaborativa a partir de un diseño tecnopedagógico, usando SMILE". Cada uno de esos trabajos muestran cómo es posible realizar diseños tecnopedagógicos para potenciar la labor docente y el aprendizaje de los estudiantes.

La investigación que soporta este artículo se gestó desde la Maestría en Pedagogía de las TIC de la Universidad de La Guajira y centra su propósito en analizar la práctica docente para implementar una estrategia pedagógica activa que permita resolver la necesidad educativa evidenciadas en el bajo rendimiento y apropiación de la estadística descriptiva en estudiantes del tercer semestre de Negocios Internacionales de la Universidad de La Guajira. Durante el desarrollo de la intervención se diseñó, implementó y evaluó el diseño tecnopedagogico puesto para dinamizar la interacción entre los principales actores de la práctica.

\section{METODOLOGIA}

La investigación es de tipo mixta, se integraron técnicas de análisis de los datos desde el enfoque cualitativo y cuantitativo. En cuanto a la metodología cualitativa, desde la investigación acción participativa (Balcazar, 2003), se realizó el análisis de contenido, entrevista semiestructurada, registro en diario de campo, observación directa a comportamiento y participación de los estudiantes en los encuentros sincrónicos y asincrónicos. Para lo cuantitativo, se analizaron los datos obtenidos de los registros estadísticos provenientes de las bitácoras de calificaciones de los reportes de la plataforma virtual Akumaja. 


\section{Participantes}

Para realizar este estudio se seleccionó el curso de estadística descriptiva del programa de Negocios Internacionales de la Universidad de La Guajira, por las dificultades evidenciadas en los estudiantes en el dominio de competencias matemáticas básicas, siendo esta una de las principales causas del bajo rendimiento académico; igualmente se les reconoce por ser estudiantes desmotivados y con poco interés por las estadísticas. Esta investigación se realizó específicamente en el segundo periodo académico del año 2016, en un curso de 21 estudiantes. La muestra de estudio estuvo compuesta por 14 estudiantes del sexo femenino (66.7\%) y 7 estudiantes del sexo masculino (33.3\%). Dentro de la muestra de estudio se encuentran un $20 \%$ de estudiantes en condición de repitentes.

\section{Instrumentos y análisis de datos}

Los instrumentos utilizados en esta investigación tuvieron mayor predominancia de lo cualitativo. Desde lo cuantitativo se utilizó un instrumento para medir los estilos/entradas de aprendizaje de los estudiantes y se aplicó instrumento "Test estilo de aprendizaje de VAK" y se tomaron los datos estadísticos de las entregas de los avances del proyecto en la plataforma virtual de la asignatura. Desde lo cualitativo, se utilizó la observación directa para recolectar datos sobre los progresos, los cambios y transformación de los estudiantes mientras se desarrollaba el proyecto y finalmente, se empleó un cuestionario con preguntas abiertas para hacer revisar los progresos y valoración de los estudiantes frente al proceso desarrollado. Las preguntas consignadas el formulario de valoración fueron: ¿Cuáles fueron sus aprendizajes durante el desarrollo de la asignatura? Y ¿Cómo le pareció el uso e implementación de la estrategia ABPy en la asignatura?

Los datos cuantitativos corresponden a los entregables o tareas subidas por los estudiantes en la plataforma virtual Akumaja y fueron analizados estadísticamente con la herramienta SPSS y de las respuestas consignada por los estudiantes el formulario de valoración, se realizó un análisis cualitativo de contenido con el software MAXQDA.

\section{RESULTADOS Y DISCUSIÓN}

El diseño tecnopedagógico se ejecutó con una secuencia didáctica de cuatro etapas, con una duración de 16 semanas, como se muestra en la tabla 1. Estas etapas son elaboradas y adaptadas de acuerdo con las dificultades evidenciadas por los estudiantes y su estructura va en consonancia con la guía propuesta en la estrategia pedagógica de Aprendizaje basado en Proyectos, según los pasos usados por Jalinus et al., (2017).

Tabla 1: Secuencia didáctica de etapas y actividades del diseño tecnopedagógico

\begin{tabular}{|c|c|c|c|c|}
\hline Etapas & Actividades & Duración/Fecha & Modalidad & Lugar \\
\hline \multirow{2}{*}{$\begin{array}{l}\text { Etapa 1, } \\
\text { conceptualiza } \\
\text { ción y } \\
\text { procesos }\end{array}$} & $\begin{array}{l}\text { Foro temático 1: Acercándonos a la } \\
\text { conceptualización de estadista descriptiva }\end{array}$ & 1 semana/ Ago. 2016 & $\begin{array}{l}\text { Virtual/ } \\
\text { Presencial }\end{array}$ & $\begin{array}{l}\text { Fuera/dentr } \\
\text { o de aula. }\end{array}$ \\
\hline & $\begin{array}{l}\text { Foro sobre videos elaborados por el docente } \\
\text { de los temas de clase }\end{array}$ & 2 semanas/Ago. 2016 & $\begin{array}{l}\text { Virtual/ } \\
\text { Presencial }\end{array}$ & $\begin{array}{l}\text { Fuera/dentr } \\
\text { o de aula. }\end{array}$ \\
\hline \multirow{2}{*}{$\begin{array}{l}\text { Etapa 2, } \\
\text { punto de } \\
\text { partida. }\end{array}$} & Conformación de equipos de trabajo/ roles & 1 semana/Sep. 2016 & Presencial & En aula \\
\hline & $\begin{array}{l}\text { Lluvia de ideas para la selección del tema y } \\
\text { pregunta problémica del contexto. }\end{array}$ & 1 semana/Sep. 2016 & Presencial & En aula \\
\hline \multirow{7}{*}{$\begin{array}{l}\text { Etapa 3, } \\
\text { desarrollo de } \\
\text { los proyectos. }\end{array}$} & $\begin{array}{l}\text { Configuración de sus instrumentos de } \\
\text { recolección de datos }\end{array}$ & 2 semanas/Sep. 2016 & Presencial & En aula \\
\hline & Aplicación del instrumento en contexto & 2 semanas/Oct. 2016 & $\begin{array}{l}\text { Virtual/ } \\
\text { Presencial }\end{array}$ & $\begin{array}{l}\text { Fuera de } \\
\text { aula. }\end{array}$ \\
\hline & Presentación primer avance del proyecto & 1 semana/Oct. 2016 & Presencial & En aula \\
\hline & $\begin{array}{l}\text { Tabulación y elaboración de gráficas de los } \\
\text { datos obtenidos en Microsoft Excel }\end{array}$ & $\begin{array}{l}2 \text { semanas/Oct. y } \\
\text { Nov. } 2016\end{array}$ & Presencial & En aula \\
\hline & Interpretación los datos obtenidos & 1 semana/Nov. 2016 & Presencial & En aula \\
\hline & $\begin{array}{l}\text { Redacción de las recomendaciones } \\
\text { resultados del proyecto realizado }\end{array}$ & 1semanas/Nov. 2016 & $\begin{array}{l}\text { Virtual/ } \\
\text { Presencial }\end{array}$ & $\begin{array}{l}\text { Fuera/dentr } \\
\text { o de aula. }\end{array}$ \\
\hline & Presentación informe técnico del proyecto & 1 semana/Nov. 2016 & Presencial & En aula \\
\hline $\begin{array}{l}\text { Etapa 4, } \\
\text { socialización } \\
\text { de los } \\
\text { resultados. }\end{array}$ & Socialización del proyecto final & 1 semana /Dic. 2016 & Presencial & En aula \\
\hline
\end{tabular}


En lo concerniente a esta investigación, las etapas fueron diseñadas para desarrollar las competencias matemáticas, comunicativas, digitales y estadísticas en los estudiantes de esta asignatura. Durante cada una de las etapas y actividades desarrolladas en el proyecto, el docente tomaba la parte inicial de las clases para explicar temas de la asignatura, la cual sería reforzada en la práctica con los proyectos. Para cada una de las actividades de aprendizaje se diseñaron y aplicaron rúbricas como instrumentos de evaluación, que contenían los criterios y nivel de desempeño de acuerdo con las actividades realizadas.

La secuencia inicia con la etapa de conceptualización y procesos, como se observa en la tabla 1. Esta primera etapa fue transversal. El trabajo preliminar por parte del docente incluyó la evaluación y selección de material de apoyo que sirvió como recurso para el aprendizaje según los temas del contenido del programa de la asignatura. En esta etapa se desarrollaron las actividades de aprendizaje con trabajo independiente, y grupal, con acompañamiento docente, que buscaban fortalecer las competencias comunicativas y el trabajo colaborativo. Entre las actividades realizadas, se encuentran la participación en foros, que se efectuaban a partir de la observación y análisis de videos elaborados por el docente y que además se apoyaban en consultas en bases de datos sobre los temas abordados en la asignatura.

Posteriormente, luego de aclarar algunos temas necesarios en la asignatura, se avanzó hacia la etapa 2, denominada punto de partida, como se muestra en la tabla 1, esta etapa consistió en la exposición oral del docente sobre la finalidad de la estrategia de aprendizaje, socialización de las reglas y condiciones de participación. En este punto, se definieron los grupos conformados por tres estudiantes, con sus respectivos roles y se establecieron tiempos de entrega de avances del proyecto. Los mismos fueron conformados buscando homogeneidad en cuanto a los estilos de aprendizajes, es decir, que en cada grupo hubiese un estilo de aprendizaje distinto (auditivo, visual y kinestésico), de tal manera que se complementaran. Para determinar los estilos de aprendizaje en los estudiantes se aplicó un tex de VAK. Una actividad desarrollada en esta etapa fue la selección de la pregunta problémica, que se escogió luego que ellos realizaron una lluvia de ideas. Además, en esta actividad se definieron los objetivos a alcanzar con el proyecto. Para apoyar la actividad se utilizaron herramientas tecnológicas como los motores de búsqueda para la conceptualización requerida, herramientas ofimáticas de transcripción de textos, el correo institucional para el envío de avances y la plataforma Akumaja.

Una vez seleccionados los temas, se inició la etapa 3, desarrollo de los proyectos, ver tabla 1. Es la etapa en la que los equipos de trabajo iniciaron la búsqueda, compilación, procesamiento, análisis y síntesis de la información del caso real seleccionado. Para cumplir con esta etapa los estudiantes debían seguir la estructura del proyecto propuesta por el docente, en la que se encontraban los ítems de definición del problema, justificación del proyecto y originalidad, objetivos del proyecto y variables de investigación, diseño del proyecto, esquema de contenido y organización e interpretación de la información.

Esta tercera etapa, estuvo presente a lo largo del semestre, los estudiantes ya agrupados, trabajaron de forma autónoma y fueron guiados, recibieron retroalimentación constante por el docente para la configuración de sus instrumentos de recolección de datos y la aplicación del mismo en su contexto próximo; además, luego de tener el insumo, debían trabajar en la tabulación y elaboración de gráficas de los datos obtenidos, utilizando el programa Microsoft Excel, esta herramienta brinda la posibilidad de automatizar y facilitar los procesos matemáticos. Otra actividad realizada por los estudiantes fue la interpretación de los datos obtenidos, esto sirvió para que pudieran redactar las observaciones y los resultados del proyecto realizado, durante todo este proceso se fortalecía el pensamiento crítico en los estudiantes. Después de consolidar todo el proceso, los grupos debían presentar un informe técnico donde evidenciaran todo el trabajo estadístico realizado con sus respectivas conclusiones y recomendaciones frente al tema trabajado.

La secuencia didáctica termina con la etapa 4, socialización de los resultados, ver tabla 1. Los estudiantes en esta etapa tuvieron la oportunidad de presentar los resultados obtenidos en sus proyectos, respondiendo a las preguntas de sus compañeros y del docente, la dinámica establecida permitió realizar autoevaluaciones, coevaluaciones y heteroevaluación por parte de los estudiantes y docente.

\section{Resultados datos cuantitativos}

Etapa de conceptualización y procesos. Con las actividades de esta etapa se trazó un objetivo de aprendizaje: lograr que los estudiantes se apropien de conceptos y procedimientos para los análisis estadísticos apoyados en la herramienta informática como la hoja de cálculo de Excel. Se diseñaron actividades de reflexión inicial, que se reforzaron mediante la indagación del estudiante sobre la importancia, significado y utilidad de la Estadística, para lo cual se dispuso en la plataforma Akumaja un foro temático. Con la participación en los foros, se pudo evidenciar un aumento en la participación de los estudiantes, ver tabla 2. 
Tabla 2. Participación en los foros

\begin{tabular}{|l|c|}
\hline Foros & Cantidad de visitas \\
\hline Temático 1 & 274 \\
\hline Dudas y preguntas & 1.202 \\
\hline Cierre & 187 \\
\hline Total & 1.663 \\
\hline
\end{tabular}

En los foros virtuales, los estudiantes de forma individual realizaron sus aportes y en algunos casos plantearon preguntas referentes a la temática tratada, con el fin de buscar en el docente 0 en sus compañeros respuesta a sus inquietudes, este espacio asincrónico facilitó la comunicación, incentivando así el aprendizaje colectivo. De la misma manera se dispusieron una serie de videotutoriales del propio docente, con contenidos sobre los procedimientos estadísticos que se pueden realizar mediante la hoja de cálculo Excel, tales como la construcción de tablas de frecuencias y cálculo de estadígrafos. El objetivo de los videos se cumplió debido a la cantidad de reproducciones, ver tabla 3 , que tuvieron y a los resultados mostrados por los estudiantes en las intervenciones durante las sesiones presenciales y virtuales.

Tabla 3. Reproducción de videos tutoriales

\begin{tabular}{|l|c|}
\hline \multicolumn{1}{|c|}{ Tutoriales } & Cantidad de Reproducción \\
\hline TDFVC & 105 \\
\hline TDFVD & 71 \\
\hline Estadígrafos de posición & 45 \\
\hline Cómo construir un cuadro resumen & 22 \\
\hline Estenógrafos de dispersión & 18 \\
\hline
\end{tabular}

El video tutorial tablas de distribución de frecuencias para variables continuas (TDFVC) y tablas de distribución de frecuencias para variables discontinuas (TDFVD) fueron los de mayor número de reproducciones. Los foros y vídeos tutoriales lograron dinamizar la formación, como se muestra en la tabla 4, ya que la comunicación pasó a ser bidireccional, donde los estudiantes plantean sus dudas o preguntas y el docente o los mismos compañeros aclaraban y daban respuestas, situación que ocurría escasamente en el aula de clases cuando la formación era de corte tradicional.

Tabla 4. Cantidad de reproducciones y vistas en la plataforma de las actividades (todos los roles)

\begin{tabular}{|l|c|c|c|c|c|}
\hline Roles & Agosto/2016 & Sept/2016 & Oct/2016 & Nov/2016 & Dic/2016 \\
\hline Estudiantes & 18 & 934 & 265 & 2291 & 1005 \\
\hline Profesor & 54 & 98 & 46 & 369 & 99 \\
\hline Invitado & 23 & 1 & 3 & 58 & 0 \\
\hline Total & 95 & 1033 & 314 & 2718 & 1104 \\
\hline
\end{tabular}

Etapa de punto de partida. En esta etapa se conformaron los equipos que trabajaron los proyectos y compartieron con el docente la temática a estudiar, como se muestra en la tabla 5. Esta etapa estuvo marcada por la conformación de los grupos colaborativos, por lo cual el docente categorizó previamente los estilos de aprendizaje de los estudiantes. Para lograr esta clasificación aplicó el modelo de prueba de estilos de aprendizaje en línea (modelo PNL) (Seitova et al., 2016). El test de VAK se aplicó para descubrir la tendencia que los estudiantes utilizan como método para aprender y así formar grupos que se pudieran complementar, sabiendo que se tienen tres sistemas para representar mentalmente la información: visual, auditivo y kinestésico. Se obtuvo que el $64 \%$ de los estudiantes tiene un estilo de aprendizaje auditivo, el $24 \%$ visual y un 14\% kinestésico, con los cuales se conformaron los 6 grupos colaborativos, teniendo en cuenta que en cada grupo estuviera integrado por estudiantes con estilo de aprendizaje distintos.

Etapa de desarrollo del proyecto. Los resultados arrojados en esta etapa mostraron como el uso de la hoja de cálculo Excel permitió que los estudiantes lograran desarrollar los procesos de tabulación y cálculo de estadígrafo. Con el uso de la herramienta, los estudiantes pudieron fortalecerse en los cálculos matemáticos que tanto les complican las operaciones estadísticas. En ese sentido, los estudiantes asumieron la responsabilidad en su proceso de aprendizaje, lo cual se constituye en un elemento necesario para que el ABPy tenga éxito. Por observación directa a la participación y comportamiento de los estudiantes en clase, se podía evidenciar como la implementación de esta nueva forma de orientar la asignatura influyó para que en los estudiantes se despertara un mayor interés y disposición al trabajo en equipo y aprender los temas de estadística I. Etapa de socialización de los resultados. En esta etapa se socializaron los proyectos que se realizaron a lo largo de la asignatura, que fueron evaluados por rúbricas preparadas por el docente. 
Tabla 5. Ideas de proyecto y conformaciones de equipos por estilos de aprendizaje.

\begin{tabular}{|c|c|c|}
\hline No & Tema & Entradas de aprendizaje \\
\hline \multirow[t]{3}{*}{1} & \multirow{3}{*}{$\begin{array}{l}\text { ¿Qué tan avanzado ha sido el desarrollo } \\
\text { de la universidad de la Guajira desde su } \\
\text { inicio hasta el día de hoy? }\end{array}$} & Visual - Auditivo \\
\hline & & Auditivo - Visual \\
\hline & & Auditivo kinestésico \\
\hline \multirow[t]{4}{*}{2} & \multirow{4}{*}{$\begin{array}{l}\text { ¿Cuál es la capacidad que tienen los } \\
\text { estudiantes para pagar el transporte a la } \\
\text { universidad? }\end{array}$} & Auditivo - Kinestésico \\
\hline & & Auditivo - Kinestésico \\
\hline & & Kinestésico - Auditivo \\
\hline & & Visual - Auditivo \\
\hline \multirow[t]{3}{*}{3} & \multirow{3}{*}{$\begin{array}{l}\text { Aceptación del nuevo restaurante de la } \\
\text { universidad de La Guajira }\end{array}$} & Auditivo - Visual \\
\hline & & Auditivo - Visual \\
\hline & & Auditivo - Kinestésico \\
\hline \multirow[t]{4}{*}{4} & \multirow{4}{*}{$\begin{array}{l}\text { Calidad de servicio en los establecimientos } \\
\text { de comercio ubicados en el centro } \\
\text { comercial Viva Wuajira. }\end{array}$} & Kinestésico - Auditivo \\
\hline & & Visual - auditivo \\
\hline & & Auditivo - Visual \\
\hline & & Auditivo - Visual \\
\hline \multirow[t]{3}{*}{5} & \multirow{3}{*}{$\begin{array}{l}\text { Análisis del porqué los estudiantes de } \\
\text { contaduría escogieron la universidad de La } \\
\text { Guajira jornada matinal para estudiar }\end{array}$} & Visual - Auditivo \\
\hline & & Auditivo - Visual \\
\hline & & Auditivo - Kinestésico \\
\hline \multirow[t]{4}{*}{6} & \multirow{4}{*}{$\begin{array}{l}\text { ¿Qué piensan y opinan los estudiantes con } \\
\text { respecto al cese de actividades en la } \\
\text { universidad de la Guajira? }\end{array}$} & Auditiva - Kinestésico \\
\hline & & Kinestésico - Auditivo \\
\hline & & Auditivo - Visual \\
\hline & & Visual - Auditivo \\
\hline
\end{tabular}

\section{Resultados datos cualitativos}

Al finalizar el proceso formativo, se aplicó a los estudiantes un formato de valoración con el propósito de conocer las impresiones finales sobre la estrategia implementada en la asignatura, ver tabla 6 . De esta valoración final se analizaron con el software MAXQDA de donde se construyen 8 categorías para el análisis, entre las que se destacan: Uso y apropiación de la ABPy, Fortalecimiento del aprendizaje, Trabajo colaborativo, Uso de TIC para el aprendizaje, Aplicación de la teoría y conocimientos, Metodología Activa, Uso y apropiación de Microsoft Excel, Comprensión con sentido de la estadística, de lo desarrollado en la asignatura, en la tabla 6, se muestran algunas valoraciones de los estudiantes.

Tabla 6. Valoración individual de los estudiantes a la metodología implementada en la asignatura

\begin{tabular}{|c|c|}
\hline Valoración de los estudiantes & Categorías de análisis \\
\hline $\begin{array}{l}\text { "A pesar de que soy repitiente de esta asignatura, puedo decirle que he sentido la } \\
\text { diferencia en cuanto al aprendizaje ya que con el profesor anterior no cumplí con esta } \\
\text { meta, todas las clases fueron complicadas, pero gracias a la ayuda de las TIC y su } \\
\text { método para enseñar estadística he aprendido mucho en comparación a mi semestre } \\
\text { anterior. Cabe resaltar que además de aplicarla en el proyecto llevándola a la vida real, } \\
\text { sé que más adelante nos servirá para otros estudios de nuestra carrera, sobre todo el } \\
\text { aprendizaje que se dio en los grupos conformados". }\end{array}$ & $\begin{array}{l}\text { Uso y apropiación de la } \\
\text { ABPy } \\
\text { Fortalecimiento del } \\
\text { aprendizaje } \\
\text { Trabajo colaborativo }\end{array}$ \\
\hline $\begin{array}{l}\text { "Primeramente impresionada por la metodología del curso, al utilizar las TIC como } \\
\text { ayuda de aprendizaje, tanto en actividades como en examen virtuales. Esto ayuda a } \\
\text { nosotros como estudiantes a ser experimentados a la hora de realizar en otra } \\
\text { oportunidad estudios virtuales. Desde un comienzo explicó que la estadística era difícil } \\
\text { de aprender, pero su metodología de explicar por medio de historias hace que el } \\
\text { estudiante analice la teoría y le sea fácil aplicarla por medio de la práctica". }\end{array}$ & $\begin{array}{l}\text { Uso de TIC para el } \\
\text { aprendizaje } \\
\text { Aplicación de la teoría y } \\
\text { conocimientos }\end{array}$ \\
\hline $\begin{array}{l}\text { "La metodología fue práctica, ya que cuando estemos laborando no vamos a realizar los } \\
\text { procedimientos en el cuaderno, allá lo tenemos que hacer en el computador y en la } \\
\text { metodología utilizada lo hacíamos en Excel, además lo hacíamos en la práctica con los } \\
\text { proyectos, es decir no nos quedábamos en la teoría, lo aplicábamos. Nosotros nunca } \\
\text { habíamos visto una asignatura así (con esa metodología) donde se practique la teoría, } \\
\text { con usted se va a la vida real, porque en las otras asignaturas, tanta teoría que a veces } \\
\text { uno no sabe para qué es, mientras que en esta sabíamos para qué es, para qué nos } \\
\text { sirve la estadística" }\end{array}$ & $\begin{array}{l}\text { Metodología Activa } \\
\text { Uso y apropiación de } \\
\text { Microsoft Excel } \\
\text { Comprensión con }\end{array}$ \\
\hline
\end{tabular}


Los resultados de las evaluaciones de los aprendizajes arrojados por la aplicación de las rúbricas dan cuenta de un mayor dominio de los contenidos de la disciplina, demostrada no solo en el progreso del manejo de los datos estadísticos contextualizados en las áreas seleccionadas, sino también en el desempeño de los estudiantes. Este proceso sistematizado en etapas generó el conocimiento y los productos esperados basados en fundamentos teóricos, metodológicos y prácticos de la Estadística Descriptiva y de acuerdo con los principios que están asociados a la metodología del aprendizaje basado en proyectos.

Tabla 7. Valoraciones de actividades realizadas en el ABPy

\begin{tabular}{|l|l|c|c|}
\hline \multirow{2}{*}{$\begin{array}{l}\text { Nombres de las } \\
\text { actividades }\end{array}$} & Genero & Masculino & Femenino \\
\cline { 2 - 4 } & Valoraciones & Porcentaje (\%) & Porcentaje (\%) \\
\hline \multirow{4}{*}{$\begin{array}{l}\text { Participación en } \\
\text { Foros }\end{array}$} & Excelente & $9,5 \%$ & $38,1 \%$ \\
\cline { 2 - 4 } & Sobresaliente & $4,8 \%$ & $14,3 \%$ \\
\cline { 2 - 4 } & Aceptable & & $4,8 \%$ \\
\cline { 2 - 4 } & Insuficiente & $19,0 \%$ & $9,5 \%$ \\
\hline \multirow{4}{*}{$\begin{array}{l}\text { Sustentación del } \\
\text { proyecto final }\end{array}$} & Excelente & $9,50 \%$ & $28,60 \%$ \\
\cline { 2 - 4 } & Sobresaliente & $19 \%$ & $28,60 \%$ \\
\cline { 2 - 4 } & Aceptable & & $9,50 \%$ \\
\cline { 2 - 4 } & Insuficiente & $4,80 \%$ & \\
\hline
\end{tabular}

En la tabla 7 se muestran las valoraciones de algunas de las actividades de aprendizaje realizadas durante el ABPy; en los foros el $71.5 \%$ de los estudiantes hicieron sus aportes y obtuvieron notas aprobatorias con valoraciones entre aceptable, sobresaliente y excelente; el género femenino tuvo un mejor y mayor desempeño en esta actividad. Por otra parte, se muestra en la tabla 7, que, en la sustentación del proyecto final, ambos géneros alcanzaron en mayor proporción notas aprobatorias y con ello se logró evidenciar el desarrollo de competencias estadísticas, comunicativas, digitales y pensamiento crítico en los estudiantes de la asignatura de estadística descriptiva. En estas actividades las rubricas de evaluación que se presentaban a los estudiantes antes de presentar sus entregables, fueron muy relevantes porque les permitieron afinar su sentido de autoevaluación frente a sus producciones.

La estrategia de ABPy soportada en el diseño tecnopedagógico presentado, es pertinente para desarrollarse en cualquier proceso de enseñanza de las diferentes áreas del saber diferentes a la estadística, como se muestra en el artículo" Aprendizaje Basado en Proyectos a través de las TIC. Una Experiencia de Innovación Docente desde las Aulas Universitarias", presentado por Ausín et al., (2016), en que se logró "resultados que muestran un alto grado de satisfacción con la creación de una radio educativa y con la utilidad educativa del proyecto". En ese sentido, los autores validan que el diseño tecnopedagógico debe ser pensado en función de las necesidades del aula y su propio contexto, los cuales permitirán que se logre una adecuada selección de la estrategia pedagógica, en este caso el ABPy y las TIC apropiadas para mediar en la enseñanza.

\section{CONCLUSIONES}

A partir de los resultados obtenidos, se pueden extraer las siguientes conclusiones: 1) Con la implementación de la metodología de aprendizaje basado en proyectos, se potenció la construcción de conocimiento en los contenidos de la asignatura Estadística I y se fortalecieron habilidad para los análisis estadísticos logrados por la población de estudiantes del curso investigado; 2) El ABPy apoyado en el diseño tecnopedagógico incrementó la capacidad de análisis y síntesis en los estudiantes; y permitió la adquisición de nuevas habilidades para el uso de las tecnologías a favor del aprendizaje; 3) Los estudiantes aprendieron a autoevaluarse de forma responsable su propio trabajo y el desempeño de sus compañeros. En la entrega del producto final, se impulsó el desarrollo de aptitudes y habilidades para el trabajo investigativo ya que se logró avanzar en el conocimiento que incluso es útil para otras asignaturas y requisitos académicos; 4) Se logró minimizar la brecha entre la teoría y la práctica en el área de conocimiento de la Estadística Descriptiva por medio de la elaboración de proyectos para encontrar soluciones a problemas en contextos reales; 5) El objetivo propuesto en este proyecto se logró con la implementación de la estrategia ABPy, lo cual ayudó a un cambió del paradigma de una metodología tradicional y unidireccional a una interacción bidireccional; en el que el estudiante conscientemente obtuvo un aprendizaje más profundo. 


\section{AGRADECIMIENTOS}

Los autores agradecen a la Universidad de La Guajira, por el apoyo brindado para la publicación de los resultados de esta investigación.

\section{REFERENCIAS}

Acharya, S., y Gayana, M. N., Enhanced learning and improved productivity of students' using project based learning approaches for programming courses, Journal of Engineering Education Transformations, 34, 524-530 (2021)

Ausín, V., Abella, V., Delgado, V., y Hortigüela, D., Aprendizaje basado en proyectos a través de las TIC, una experiencia de innovación docente desde las aulas universitarias. http://dx.doi.org/10.4067/S0718-50062016000300005, Formación Universitaria, 9(3), 31-38 (2016)

Balcazar, F.E., Investigación acción participativa (iap): aspectos conceptuales y dificultades de implementación, Fundamentos en Humanidades, 4(7-8), 59-77 (2003)

Batanero, C., Díaz, C., Contreras, J. M., y Roa, R., El sentido estadístico y su desarrollo, Números: Revista de Didáctica de las Matemáticas, 83, 7-18, (2013)

Berger, C., y Kam, R., Definitions of instructional design, adapted "Training and Instructional Design", Applied Research Laboratory, Penn State University, 26 (05) (1996)

Burgos-Leiva, C. A., Rementeria-Piñones, J. A., Espinoza-Oyarzún, J. C., y Rodríguez-García, A. B., Aprendizaje basado en proyectos aplicados en la asignatura de materiales de construcción. https://dx.doi.org/10.4067/S071850062021000200105, Formación Universitaria, 14(2), 105-112, (2021)

Clark, R. M., Wang, M., Splain, Z. A., y Chen, K. P., Teaching a standalone optics and lasers course using project-based learning, IEEE Transactions on Education, 63(4), 255-262 (2020)

Cruz, R. L., Serrano, C. L., y Rodríguez, B. J., Modelo de mejoramiento productivo: una aplicación de la fabricación digital incorporada al aprendizaje basado en proyectos (ABP) en la educación superior,

https://dx.doi.org/10.4067/S0718-50062021000200065, Formación Universitaria, 14(2), 65-74, (2021)

Dierker, L., Evia, J. R., y otros 5 autores, Project-based learning in introductory statistics: comparing course experiences and predicting positive outcomes for students from diverse educational settings, International Journal of Educational Technology and Learning, 3(2), 52-64 (2018)

Flores, A., y Pinto, J., Características de la enseñanza de la estadística por proyectos, Acta Latinoamericana de Matemática Educativa 30, 263-271 (2017)

Gonzalez-Ramirez, T., y Pedraza-Navarro, Social and families variables associated with university drop-out. Educatio Siglo XXI, 35(2), 365-387 (2017)

Gutiérrez-Monsalve, J. A., Garzón, J., y Segura-Cardona, A. M., Factores asociados al rendimiento académico en estudiantes universitarios, https://dx.doi.org/10.4067/S0718-50062021000100013, Formación Universitaria, 14(1), 13-24 (2021)

Gutiérrez Tapias, M., Estilos de aprendizaje, estrategias para enseñar, su relación con el desarrollo emocional y aprender a aprender, https://doi.org/10.15366/tp2018.31.004, Tendencias pedagógicas. 31, 83-96 (2018)

Herrera, R. F., Collaborative project-based learning of environments programming from civil engineering projects, https://doi.org/10.15359/ree.21-2.10, Revista Electrónica Educare, 21(2), 205-222 (2017)

Jalinus, N., Nabawi, R. A., y Mardin, A., The seven steps of project based learning model to enhance productive competences of vocational students, Atlantis Press, 251-256 (2017)

Kokotsaki, D., Menzies, V., Wiggins, A., Project-based learning: a review of the literature, https://doi.org/10.1177/1365480216659733, Improving Schools, 19 (3), 267-277 (2016)

Kokotsaki, D., Menzies, V., y Wiggins, A., Project-based learning: a review of the literature, Improving Schools, 19(3), 267-277 (2016)

Leung, A., Exploring techno-pedagogic task design in the mathematics classroom, in digital technologies in designing mathematics education tasks, 3-16, Springer, Cham, (2017)

Mateos, E., Redondo, S., y otros tres autores, Implantación de una metodología docente activa en la asignatura de redacción y ejecución de proyectos del grado en biología, http://dx.doi.org/10.21703/rexe.20201939mateos15, Revista de estudios y experiencias en educación, 19(39), 259-274 (2020)

Rodríguez, B., Ordoñez, M., y Meneses, L., Strengthening of reasoning levels in higher education students through the use of learning strategies (problem-based learning and collaborative learning) using ICT'S, Electronic Journal of Research in Educational Psychology, 16(2) 477-502 (2018)

Seitova, S. M., Kozhasheva, G. O., y otros 5 autores., Peculiarities of using neuro-linguistic programming techniques in teaching, International Electronic Journal of Mathematics Education, 11(5), 1135-1149 (2016) 
Serrano, V. C., y Blasco, O. B., El uso de gráficos interactivos en Excel para facilitar la comprensión de conceptos básicos de estadística @ tic, Revista D'Innovació Educativa, (5), 30-34 (2010)

Solano, A D., y Aarón, M. A., Enseñanza en ingeniería de manera colaborativa a partir de un diseño tecnopedagógico, usando SMILE, https://dx.doi.org/10.4067/S0718-50062020000400201, Formación Universitaria, 13(4), 201-210 (2020)

Souza, M., Zea, A., Rodríguez, G., y Molina, A., Estilo de vida y factores socioeconómicos en estudiantes de electivas de actividad física y deporte de la Pontificia Universidad Javeriana, Análisis, 49(90), 229-243 (2017)

Travieso, D., y Ortiz, T., Aprendizaje basado en problemas y enseñanza por proyectos: alternativas diferentes para Enseñar, Revista Cubana de Educación Superior, 37(1), 124-133 (2018)

Veselov, G. E., Pljonkin, A. P., y Fedotova, A. Y., Project-based learning as an effective method in education, In Proceedings of the 2019 International Conference on Modern Educational Technology, 54-57 (2019)

Villalobos-Abarca, M., Herrera-Acuña, R., y otros dos autores, Aprendizaje basado en proyectos reales aplicado a la formación del ingeniero de software, http://dx.doi.org/10.4067/S0718-50062018000300097, Formación Universitaria, 11(3), 97-112 (2018)

Yeh, Y. F., Hsu, Y. S., Wu, H. K., y Chien, S. P., Exploring the structure of TPACK with video-embedded and disciplinefocused assessments, https://doi.org/10.1016/j.compedu.2016.10.006, Computers and Education, 104, 49-64 (2017) 